\title{
Numerical simulation of predicting and reducing solid particle erosion of solid-liquid two-phase flow in a choke
}

\author{
Li Guomei ${ }^{1,2}$, Wang Yueshe ${ }^{*}$, He Renyang ${ }^{2}$, Cao Xuewen ${ }^{3}$, Lin Changzhi ${ }^{4}$ \\ and Meng Tao ${ }^{2}$ \\ ${ }^{1}$ State Key Laboratory of Multiphase Flow in Power Engineering, Xi'an Jiaotong University, Xi'an, Shaanxi 710049, China \\ ${ }^{2}$ China Special Equipment Inspection and Research Institute, Beijing 100013, China \\ ${ }^{3}$ School of Transport \& Storage and Civil Engineering, China University of Petroleum, Qingdao, Shandong 266555, China \\ ${ }^{4}$ Petroleum Exploration and Production Research Institute, Sinopec, Beijing 100083, China
}

\begin{abstract}
Chokes are one of the most important components of downhole flow-control equipment. The particle erosion mathematical model, which considers particle-particle interaction, was established and used to simulate solid particle movement as well as particle erosion characteristics of the solid-liquid two-phase flow in a choke. The corresponding erosion reduction approach by setting ribs on the inner wall of the choke was advanced. This mathematical model includes three parts: the flow field simulation of the continuous carrier fluid by an Eulerian approach, the particle interaction simulation using the discrete particle hard sphere model by a Lagrangian approach and calculation of erosion rate using semiempirical correlations. The results show that particles accumulated in a narrow region from inlet to outlet of the choke and the dominating factor affecting particle motion is the fluid drag force. As a result, the optimization of rib geometrical parameters indicates that good anti-erosion performance can be achieved by four ribs, each of them with a height $(H)$ of $3 \mathrm{~mm}$ and a width $(B)$ of $5 \mathrm{~mm}$ equaling the interval between ribs $(L)$.
\end{abstract}

Key words: Solid-liquid two-phase flow, discrete particle hard sphere model, choke, erosion rate, antierosion, numerical simulation

\section{Introduction}

The mechanical damage to the surface caused by the impact of solid particles has been a serious problem in a variety of engineering applications. Any industrial process involving the transportation of solid particles entrained in the fluid phase can be subject to erosion damage (Humphrey, 1990; Finnie, 1995). In oil and gas production, the solid particles, which were used as proppants and carried by fracturing fluids with a high velocity in sand fracturing operations, can cause serious damage to downhole flowcontrol equipment (chokes) as well as the surface of casing walls (Economides and Nolte, 2002; McCasland et al, 2004; Vincent et al, 2004). During oil-field water injection operations, particles entrained in continuous fluids can also cause damage to the casing wall (Jordan, 1998; Richardson et al, 1986). Depending on the actual conditions, the erosion damage may be severe and extremely expensive, as it may be frequently necessary to replace or repair the device or component that is exposed and susceptible to the erosive environment (McCasland et al, 2004; Jordan 1998; McLaury

* Corresponding author. email: wangys@mail.xjtu.edu.cn

Received August 25, 2008 et al, 1996; Wallace et al, 2004). Hence, it becomes more and more important to predict the erosion caused by particle impact accurately and to develop corresponding erosion reduction methods.

Computational Fluid Dynamics (CFD) has been used in the research on solid particle erosion for many years. CFD-based erosion prediction process includes several different aspects (Zhang, 2006): flow modeling, particlefluid interaction, particle-particle interaction, particle-wall interaction and particle erosion modeling. Each aspect by itself is very complex, and many researchers have made great efforts in order to better understand the mechanisms. The current erosion computational models are established based on different mechanisms, which show satisfactory application to predicting particle movement characteristics, calculating the erosion rate of wall surfaces and improving particle tracking in order to reduce erosion (Chen et al, 2006; Fan et al, 2004; Forder et al, 1998; Habib and Badr, 2004; McCasland et al, 2004; McLaury and Wang 1997; Song et al, 1996; Yao et al, 2002). However, most of these models neglect the influence of particle movement on the fluid as well as particle-particle interactions. These are one-way coupling methods and only applicable to the conditions of low volume fraction of the discrete particle phase. The four-way coupling 
computational model (Elghobashi, 1994), which takes particle-fluid interaction and particle-particle interaction into consideration at the same time, has been rarely reported in the literature, especially in the study of erosion of well pipeline systems.

The purpose of this paper is to provide deeper understanding of solid particle erosion characteristics in the choke and to change the flow field geometry in order to reduce erosion. In this paper, the discrete particle hard sphere model was used to simulate particle-particle interaction; after obtaining information on particle movement (impact velocity and impact angle), the semi-empirical correlations were used to calculate particle erosion rate. Fortunately, this model not only takes particle-fluid interaction into consideration but particle-particle interaction as well, and is a four-way coupling method. Therefore, this model avoids the shortcomings of former models mentioned above and is applicable to the case of high volume fractions of the particle phase.

\section{Mathematical model}

The mathematical model includes the following three parts, namely the continuous carrier fluid flow field simulation by an Eulerian approach, particle-particle interaction simulation using a discrete particle hard sphere model by a Lagrangian approach, and erosion calculation using semiempirical correlations.

\subsection{Governing equations of continuous flow}

The equations of continuous flow are derived from the volume averaged Navier-Stokes equations, which take into account the influence of fluid volume fraction and drag force between the fluid and particle phases. The continuity and momentum equations are expressed as follows:

$$
\begin{aligned}
& \frac{\partial}{\partial t}\left(a_{\mathrm{f}} \rho_{\mathrm{f}}\right)+\nabla \cdot\left(a_{\mathrm{f}} \rho_{\mathrm{f}} \boldsymbol{u}_{\mathrm{f}}\right)=0 \\
& \frac{\partial}{\partial t}\left(a_{\mathrm{f}} \rho_{\mathrm{f}} \boldsymbol{u}_{\mathrm{f}}\right)+\nabla \cdot\left(a_{\mathrm{f}} \rho_{\mathrm{f}} \boldsymbol{u}_{\mathrm{f}} \boldsymbol{u}_{\mathrm{f}}\right) \\
& =-a_{\mathrm{f}} \nabla p+\nabla \cdot\left(a_{\mathrm{f}} \tau_{\mathrm{f}}\right)+a_{\mathrm{f}} \rho_{\mathrm{f}} \boldsymbol{g}-\boldsymbol{f}_{\text {drag }}
\end{aligned}
$$

where $\rho_{\mathrm{f}}, \boldsymbol{u}_{\mathrm{f}}$ and $p$ are the fluid density, velocity, and pressure, respectively; $\boldsymbol{g}$ is gravity acceleration; $\tau_{\mathrm{f}}$ is the fluid shear stress; $a_{\mathrm{f}}$ is the volume fraction of fluid; and $\boldsymbol{f}_{\mathrm{drag}}$ is volumetric fluid-particle interaction force, which can be given as:

$$
\begin{aligned}
& a_{\mathrm{f}}=1-\sum_{i=1}^{n} V_{\mathrm{p} i} / \Delta V \\
& \boldsymbol{f}_{\mathrm{drag}}=\frac{1}{\Delta V} \sum_{i=1}^{n} \boldsymbol{F}_{\mathrm{drag}, i}
\end{aligned}
$$

where $\Delta V$ and $V_{\mathrm{p} i}$ are the volume of a computational cell and the volume of particle $i$ inside this cell, respectively; $\boldsymbol{F}_{\text {drag }}$ is the fluid drag force for an individual particle; $n$ is the number of particles in the cell.

The flow is turbulent, and the fluid turbulence is treated with the standard $k-\varepsilon$ turbulent model.

\subsection{Discrete particle hard sphere model}

\subsubsection{Inter-particle collision model}

Inter-particle collision is described by a hard sphere model. The hard sphere model is based on binary quasiinstantaneous collisions. It neglects particle deformation during collision, resulting in a high calculation efficiency (Crowe et al, 1998).

Collision of two particles can be shown in Fig. 1. All the post-collision velocities can be expressed as follows:

$$
\begin{aligned}
& \boldsymbol{v}_{1}=\boldsymbol{v}_{1}^{0}-\left(\boldsymbol{n}+\mu_{\mathrm{s}} \boldsymbol{t}\right)\left(\boldsymbol{n} \cdot \boldsymbol{G}^{0}\right)(1+e) \frac{m_{2}}{m_{1}+m_{2}} \\
& \boldsymbol{v}_{2}=\boldsymbol{v}_{2}^{0}+\left(\boldsymbol{n}+\mu_{\mathrm{s}} \boldsymbol{t}\right)\left(\boldsymbol{n} \cdot \boldsymbol{G}^{0}\right)(1+e) \frac{m_{1}}{m_{1}+m_{2}} \\
& \omega_{1}=\omega_{1}^{0}-\frac{5}{2 R_{1}}\left(\boldsymbol{n} \cdot \boldsymbol{G}^{0}\right)(\boldsymbol{n} \times \boldsymbol{t}) \mu_{\mathrm{s}}(1+e) \frac{m_{2}}{m_{1}+m_{2}} \\
& \omega_{2}=\omega_{2}^{0}-\frac{5}{2 R_{2}}\left(\boldsymbol{n} \cdot \boldsymbol{G}^{0}\right)(\boldsymbol{n} \times \boldsymbol{t}) \mu_{\mathrm{s}}(1+e) \frac{m_{1}}{m_{1}+m_{2}}
\end{aligned}
$$

for the case where the two spheres slide, and

$$
\begin{aligned}
& \boldsymbol{v}_{1}=\boldsymbol{v}_{1}^{0}-\left[(1+e)\left(\boldsymbol{n} \cdot \boldsymbol{G}^{0}\right) \boldsymbol{n}+\frac{2}{7}\left|\boldsymbol{G}_{\mathrm{ct}}^{0}\right| \boldsymbol{t}\right] \frac{m_{2}}{m_{1}+m_{2}} \\
& \boldsymbol{v}_{2}=\boldsymbol{v}_{2}^{0}+\left[(1+e)\left(\boldsymbol{n} \cdot \boldsymbol{G}^{0}\right) \boldsymbol{n}+\frac{2}{7}\left|\boldsymbol{G}_{\mathrm{ct}}^{0}\right| \boldsymbol{t}\right] \frac{m_{1}}{m_{1}+m_{2}} \\
& \omega_{1}=\omega_{1}^{0}-\frac{5}{7 R_{1}}\left|\boldsymbol{G}_{\mathrm{ct}}^{0}\right|(\boldsymbol{n} \times \boldsymbol{t}) \frac{m_{2}}{m \boldsymbol{m}+m_{2}} \\
& \boldsymbol{\omega}_{2}=\omega_{2}^{0}-\frac{5}{7 R_{2}}\left|\boldsymbol{G}_{\mathrm{ct}}^{0}\right|(\boldsymbol{n} \times \boldsymbol{t}) \frac{m_{1}}{m_{1}+m_{2}}
\end{aligned}
$$

for the case where two spheres stop sliding during the collision process, where superscript 0 means before collision; $\mu_{\mathrm{s}}$ is fraction coefficient; $e$ is the coefficient of restitution; $\boldsymbol{n}$ is the unit normal vector from particle 1 to particle 2 at the moment of contact; $m$ is the particle mass; $R$ is particle radius; $\boldsymbol{v}$ is particle velocity; $\omega$ is angular velocity; $\boldsymbol{G}^{0}$ is the relative velocities between particle centers before collision, and $\boldsymbol{G}_{\mathrm{ct}}{ }^{0}$ is the tangential component of the relative velocity; $t$ is the unit tangential vector at contact point.

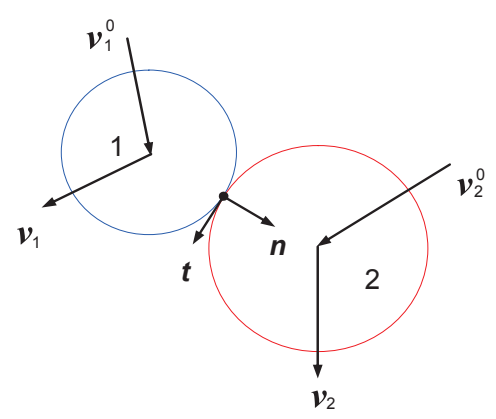

Fig. 1 Particle-particle collision

\subsubsection{Fluid drag force}

The coupling action between fluid and particle can be expressed through the fluid drag force $\boldsymbol{F}_{\text {drag }}$ of an individual particle. Using the modified fluid drag force correlation (Di Felice, 1994), the fluid drag force can be described as follows: 


$$
\boldsymbol{F}_{\text {drag }}=\frac{C_{\mathrm{d} 0}}{8} \pi d_{\mathrm{p}}^{2} \rho_{\mathrm{f}} a_{\mathrm{f}}^{2}\left|\boldsymbol{u}_{\mathrm{f}}-\boldsymbol{u}_{\mathrm{p}}\right|\left(\boldsymbol{u}_{\mathrm{f}}-\boldsymbol{u}_{\mathrm{p}}\right) a_{\mathrm{f}}^{-\chi}
$$

with

$$
\chi=3.7-0.65 \exp \left(-\frac{\left(1.5-\log R e_{\mathrm{p}}\right)^{2}}{2}\right)
$$

where $d_{\mathrm{p}}$ is the particle diameter; $\boldsymbol{u}_{\mathrm{f}}$ is the fluid velocity; $\boldsymbol{u}_{\mathrm{p}}$ is the particle velocity; $C_{\mathrm{d} 0}$ and $R e_{\mathrm{p}}$ are the fluid drag coefficient and particle Reynolds number, respectively, and they can be expressed as follows:

$$
\begin{aligned}
& C_{\mathrm{d} 0}=\left(0.63+\frac{4.8}{R e_{\mathrm{p}}^{0.5}}\right)^{2} \\
& \operatorname{Re}_{\mathrm{p}}=\frac{a_{\mathrm{f}} \rho_{\mathrm{f}} d_{\mathrm{p}}\left|\boldsymbol{u}_{\mathrm{f}}-\boldsymbol{u}_{\mathrm{p}}\right|}{\mu_{\mathrm{f}}}
\end{aligned}
$$

\subsubsection{Equation of particle motion}

The behavior of all particles is simulated by a Lagrangian approach. Because inter-particle collision is described by the hard sphere model, the inter-particle force and particle shape deformation can be neglected (Crowe et al, 1998). Thus forces acting on an individual particle mainly include gravitational and fluid drag forces during particle movement (Zhang, 2006). Other forces such as virtual mass force, pressure gradient force, Saffman lift force, Magnus lift force and Basset history force acting on a particle could be neglected in this study (Habib and Badr 2004; Habib et al, 2007; Meng and Van der Geld 1991). The motion of an individual particle is determined by Newton's second law of motion. The equation of particle can be written as follows:

$$
m_{\mathrm{p}} \frac{\mathrm{d} \boldsymbol{u}_{\mathrm{p}}}{\mathrm{d} t}=m_{\mathrm{p}} \boldsymbol{g}+\boldsymbol{F}_{\mathrm{drag}}
$$

where $m_{\mathrm{p}}$ is the particle mass.

\subsection{Semi-empirical correlations used to calculate particle erosion rate}

According to the dynamic characteristics of solid-liquid two-phase flow (which is different from gas-solid twophase flow) and the material characteristics of the choke, the calculation model proposed by Mengütürk and Sverdrup (1979) was applied in this study:

$$
\begin{aligned}
E_{\mathrm{v}}= & 1.63 \times 10^{-6}\left(\boldsymbol{W}_{1} \cos \beta_{1}\right)^{2.5} \sin \left(\frac{\pi \beta_{1}}{45.4}\right) \\
& +4.68 \times 10^{-7}\left(\boldsymbol{W}_{1} \sin \beta_{1}\right)^{2.5}, \quad \beta_{1} \leq 22.7^{\circ} \\
E_{\mathrm{v}}= & 1.63 \times 10^{-6}\left(\boldsymbol{W}_{1} \cos \beta_{1}\right)^{2.5} \\
& +4.68 \times 10^{-7}\left(\boldsymbol{W}_{1} \sin \beta_{1}\right)^{2.5}, \quad \beta_{1}>22.7^{\circ}
\end{aligned}
$$

where $E_{\mathrm{v}}$ is the particle erosion rate, $\mathrm{mm}^{3} / \mathrm{g} ; \boldsymbol{W}_{1}$ is the wall impact velocity of the solid particle; and $\beta_{1}$ is the impact angle of the solid particle.

\subsection{Criterion for occurrence of collision of two particles in the hard sphere model}

In the hard sphere model system, the initiative search approach is used to judge the binary collisions. It is supposed that the two particles $i$ and $j$ happen to collide after the time $\Delta t_{\mathrm{c}}$, so $R_{i j}=\boldsymbol{r}_{i j}+\boldsymbol{v}_{i j} \Delta t_{\mathrm{c}}$, as a result $\Delta t_{\mathrm{c}}$ can be calculated with the following expression (Kang and Guo, 2006):

$$
\Delta t_{\mathrm{c}}=\frac{-\boldsymbol{r}_{i j} \cdot \boldsymbol{v}_{i j}-\sqrt{\left(\boldsymbol{r}_{i j} \cdot \boldsymbol{v}_{i j}\right)^{2}-\left|\boldsymbol{v}_{i j}\right|^{2}\left(\left|\boldsymbol{r}_{i j}\right|^{2}-\left|R_{i j}\right|^{2}\right)}}{\left|\boldsymbol{v}_{i j}\right|^{2}}
$$

with

$$
r_{i j}=r_{i}-r_{j}, v_{i j}=v_{i}-v_{j}, R_{i j}=-\left(R_{i}+R_{j}\right) n
$$

where $\boldsymbol{r}$ is the location vector of particles.

If $\Delta t_{\mathrm{c}}<0$ or if $\boldsymbol{r}_{i j} \cdot \boldsymbol{v}_{i j}>0$, the two particles will not collide.

\section{Physical problem and simulation conditions}

The computational domain is a two-dimensional rectangular region of $0.1 \mathrm{~m} \times 0.038 \mathrm{~m}$, which is the same as the actual size of the choke, the inlet radius is $0.024 \mathrm{~m}$ and the outlet diameter is $0.018 \mathrm{~m}$, as shown in Fig. 2. The hatching boundary is a wall boundary condition, where a no-slip boundary condition is imposed for fluid flow. In the particlewall collision, the interaction of a particle with the wall is modeled in the same manner as a particle-particle collision, and the wall is considered as one particle with infinitely large diameter (Crowe et al, 1998). The top dashed axis boundary is a symmetry boundary and zero normal gradients are used for all variables. As for particles, the reflective boundary condition is used on the axis boundary. The direction of gravity goes along the $X$ direction. The fluid flows into the choke from the inlet, where a velocity inlet boundary condition is imposed for continuous flow, and the fluid flows out through the outlet, where a pressure outlet boundary condition is imposed.

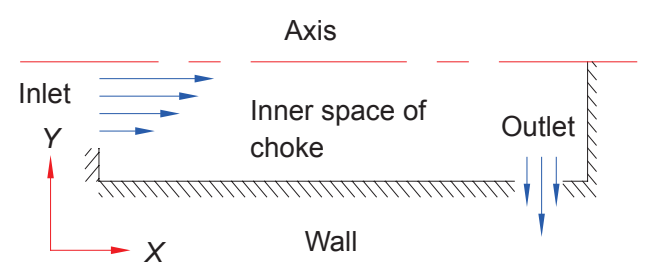

Fig. 2 Computational domain and coordinate system

The continuous fluid is water with a density of 1,000 $\mathrm{kg} / \mathrm{m}^{3}$ and a dynamic viscosity of $1.005 \times 10^{-3} \mathrm{~Pa} \cdot \mathrm{s}$. The calculation temperature is room temperature, and the flow in the choke is turbulent. The velocity at inlet section follows the $1 / 7$ power function distribution, and the turbulent center velocity was chosen as $5 \mathrm{~m} / \mathrm{s}$.

The particles all have the same diameter $(0.4 \mathrm{~mm})$ and density $\left(2,650 \mathrm{~kg} / \mathrm{m}^{3}\right)$. The friction coefficient and restitution coefficient are 0.4 and 0.9 , respectively. In the 
computational domain, there are 3,000 static initial particles located randomly. As well, another 100 particles are located uniformly at the inlet section $(X=0)$, having the same velocity as the continuous fluid cell where the particles are located. The material of the choke is carbon steel.

The computational time step for fluid is chosen as $3.0 \times$ $10^{-5} \mathrm{~s}$, and the time step for particles is $2.0 \times 10^{-6} \mathrm{~s}$. That is to say, there are 15 integration steps for particle trajectory in every time step for fluid motion.

The conventional SIMPLEC method is used to solve the governing equations for the continuous flow. The QUICK scheme is used for the convective term of the momentum equation. The explicit time integration method is used to solve the equations of discrete particles. In order to reduce the CPU time, for each particle, a neighbor list is used to store all neighboring particles, and a check for possible collisions is performed only for the particles in the list.

\section{Simulation results and discussion}

\subsection{Validation}

In order to verify the accuracy of the computational model, the present scheme is used to calculate the erosion rate of solid particles in a sudden contraction with a diameter ratio of 0.5 . The particle volumetric flow rate is $1 \mathrm{~cm}^{3} / \mathrm{s}$ and the sand diameter is $0.4 \mathrm{~mm}$. The continuous phase is water. Comparison with the results of Habib and Badr (2004) was made at different inlet velocity values as shown in Fig. 3. The results are in good agreement with the result of Habib and Badr. However, the present results are a little higher than those of Habib and Badr, which may be caused by the particleparticle interaction under turbulent condition in this study.

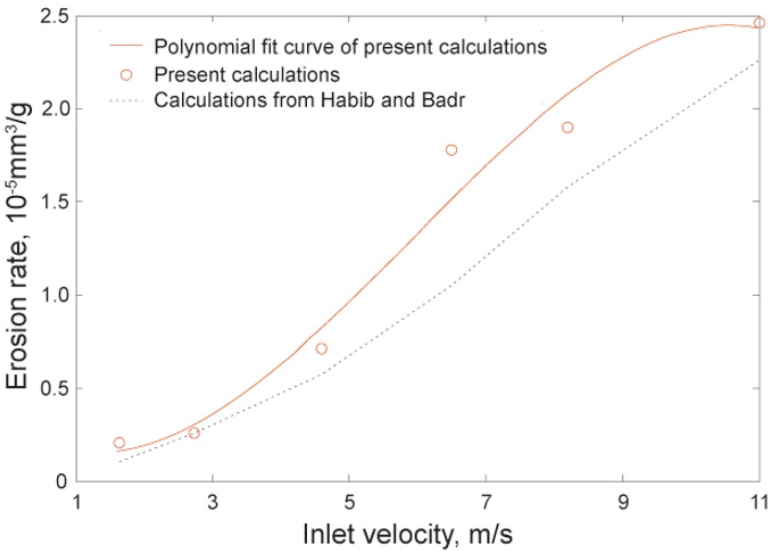

Fig. 3 Comparison of the present calculated erosion rate and the results of Habib and Badr (2004)

\subsection{Characteristics of particles' spatial and velocity distributions}

The spatial and velocity distributions of particles in the choke are shown in Fig. 4. The solid particles are carried by continuous fluid flow and move ahead, where particleparticle and particle-wall collisions will occur. Particles can be decelerated due to energy loss during particle collisions and then obtain energy from fluid flow and other particles, which leads to the particle movements in the choke reaching a dynamic steady state. Particles accumulate densely in a narrow domain from inlet to outlet of the choke when they are carried by continuous fluid flow, while particles are located sparsely near the area of axis and inlet wall. This reflects that the discrete particle hard sphere model can describe the nonuniform discrete characteristics of particle distribution in
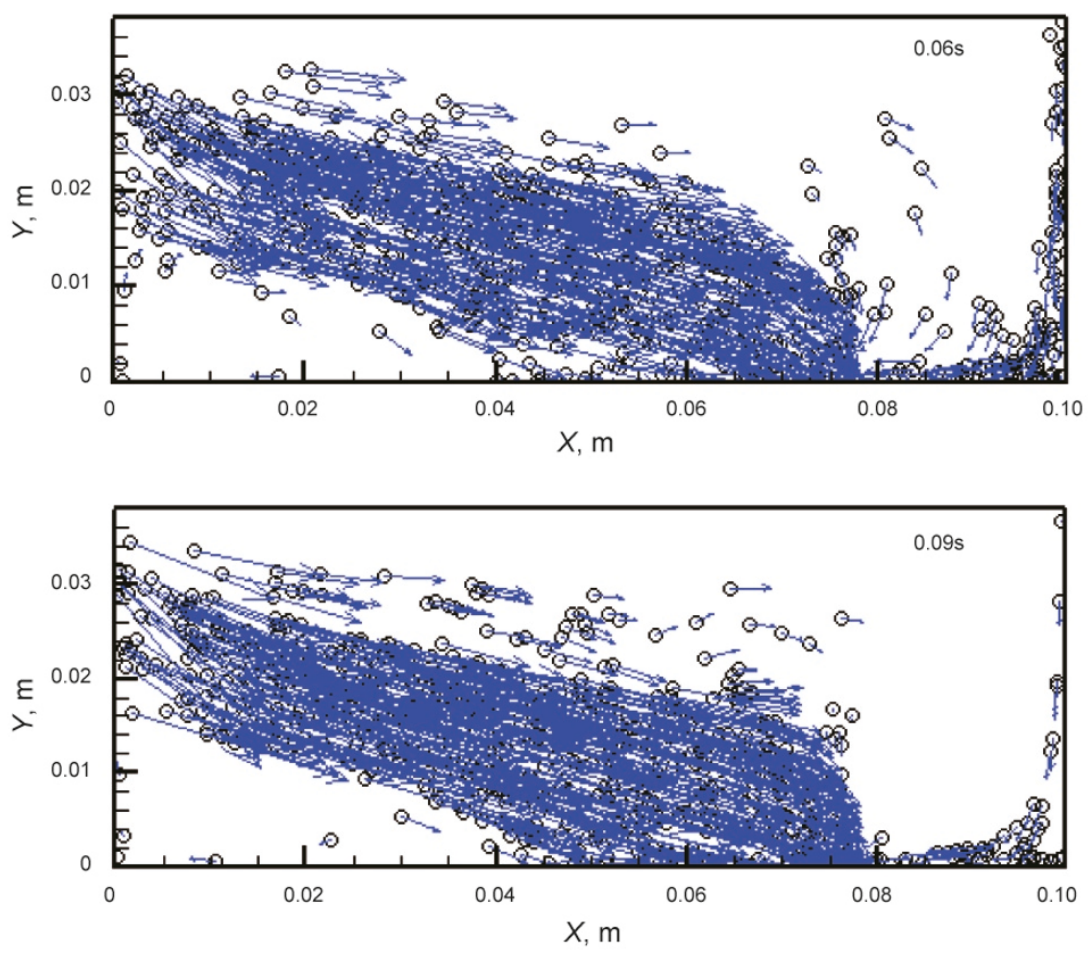

Fig. 4 Spatial and velocity distribution of particles 
the choke. On the other hand, this indicates the dominating factor affecting particle motion is the fluid drag force between particle and fluid, although there are gravitational force and acceleration force acting on particles.

The length of arrowhead in Fig. 4 means the relative magnitude of particle velocity. Therefore it can also be seen that the largest particle velocity occurs in the narrow domain from inlet to outlet. And the largest collision intensity area (namely the place where the most collisions and the largest particle velocity occur) is located in the upper and lower regions of the edge, which is near the outlet of the choke. Some particles' trajectories deviate from the streamline of continuous flow, which indicates that the particle collisions play an important role in solid-liquid two-phase movements.

\subsection{Choke geometry optimization}

According to the above-mentioned analysis, it is obvious that the most severe erosion in the choke occurs in the locations with the largest particle-particle collision intensity. However, it is most desirable for such erosive effects to be minimized. At present, the erosion reduction method, which optimizes equipment design by keeping the velocity and impact angle of particles away from the serious erosion range is a popular approach (Forder et al, 1998; McCasland et al, 2004; Song et al, 1996; Yao et al, 2002). Therefore the erosion reduction method adopted in this paper is setting several ribs with given geometry on the inner wall of the choke, in order to reduce the particle impact velocity and to change the impact angle. The sketch map of ribs is shown in Fig. 5, in which $L$ is the space between two neighboring ribs, $B$ and $H$ are width and height of ribs, respectively. The rib geometrical parameters adopted in this study are shown in Table. 1

The spatial and velocity distribution of particles in the choke after setting ribs when the calculation time is $0.09 \mathrm{~s}$ is

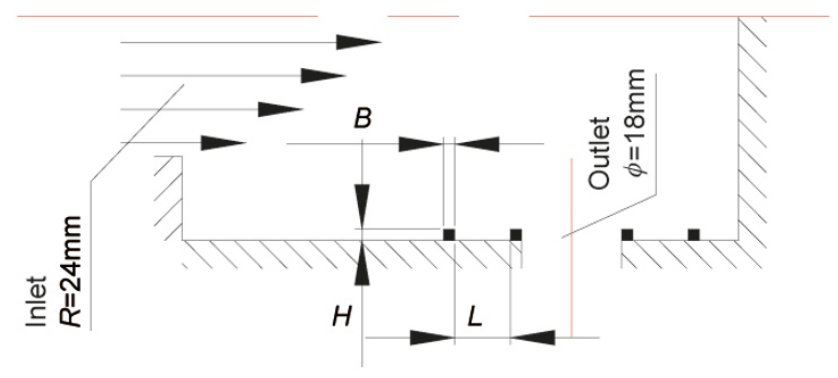

Fig. 5 Sketch map of ribs given in Fig. 6. Compared with the particle distribution in the choke before setting ribs as shown in Fig. 4, it can be easily seen that the number of particles colliding with the choke wall decreases greatly, and the impact velocity is reduced (The length of arrowhead means the relative magnitude of particle velocity as mentioned above) after setting ribs.

Table 1 The rib geometrical parameters

\begin{tabular}{ccccc}
\hline No. & $H, \mathrm{~mm}$ & $B, \mathrm{~mm}$ & $L, \mathrm{~mm}$ & Number of ribs \\
\hline O & 0 & 0 & 0 & 0 \\
A & 3 & 2 & 10 & 4 \\
B & 2 & 2 & 10 & 4 \\
C & 3 & 2 & 10 & 5 \\
D & 3 & 3 & 10 & 4 \\
E & 3 & 5 & 10 & 4 \\
F & 3 & 5 & 5 & 4 \\
G & 3 & 5 & 5 & 5 \\
H & 3 & 8 & 5 & \\
\hline
\end{tabular}

Considering the important influence of particle impact velocity on erosion rate (Finnie, 1995; Humphrey, 1990; Lindsley and Marder 1999; Zhang, 2006), the impact velocity was labeled into several sections with a $0.5 \mathrm{~m} / \mathrm{s}$ velocity interval. And then the probability distribution of particlewall impact velocity, which was defined as the percentage of wall-collision particles with velocity lying in an individual velocity interval labeled above (of all wall-collision particles) during the calculation period, was analyzed. The probability distribution of impact velocity in the choke with different rib geometrical parameters given in Table 1 is shown in Fig. 7. Fig. 7 shows that about $40 \%$ of the particles have a velocity less than $0.5 \mathrm{~m} / \mathrm{s}$ when the choke has no ribs fitted. However, more than $90 \%$ of the particles have a velocity of less than $0.5 \mathrm{~m} / \mathrm{s}$ account when the ribs are in place. That is to say that the average impact velocity of particles lies in the low impact velocity domain when ribs are used.

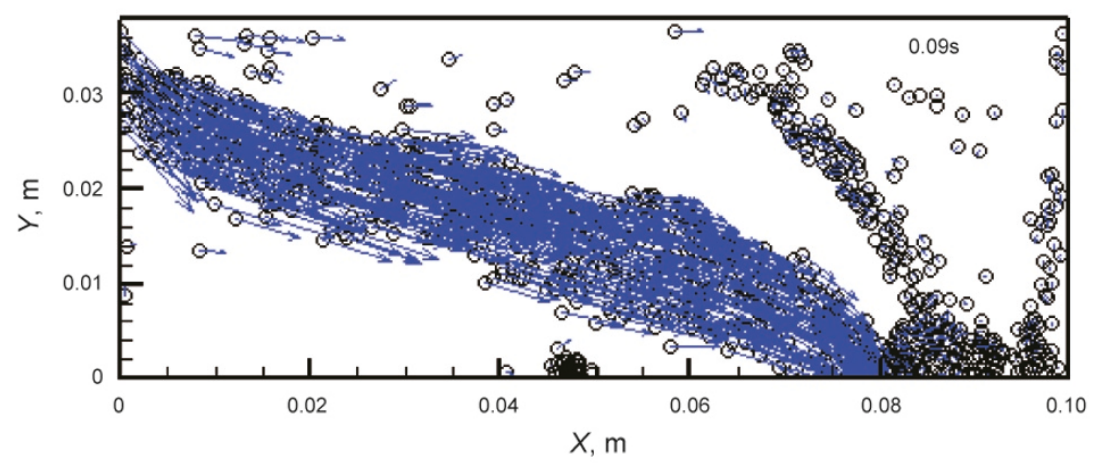

Fig. 6 Spatial and velocity distribution of particles after setting ribs 

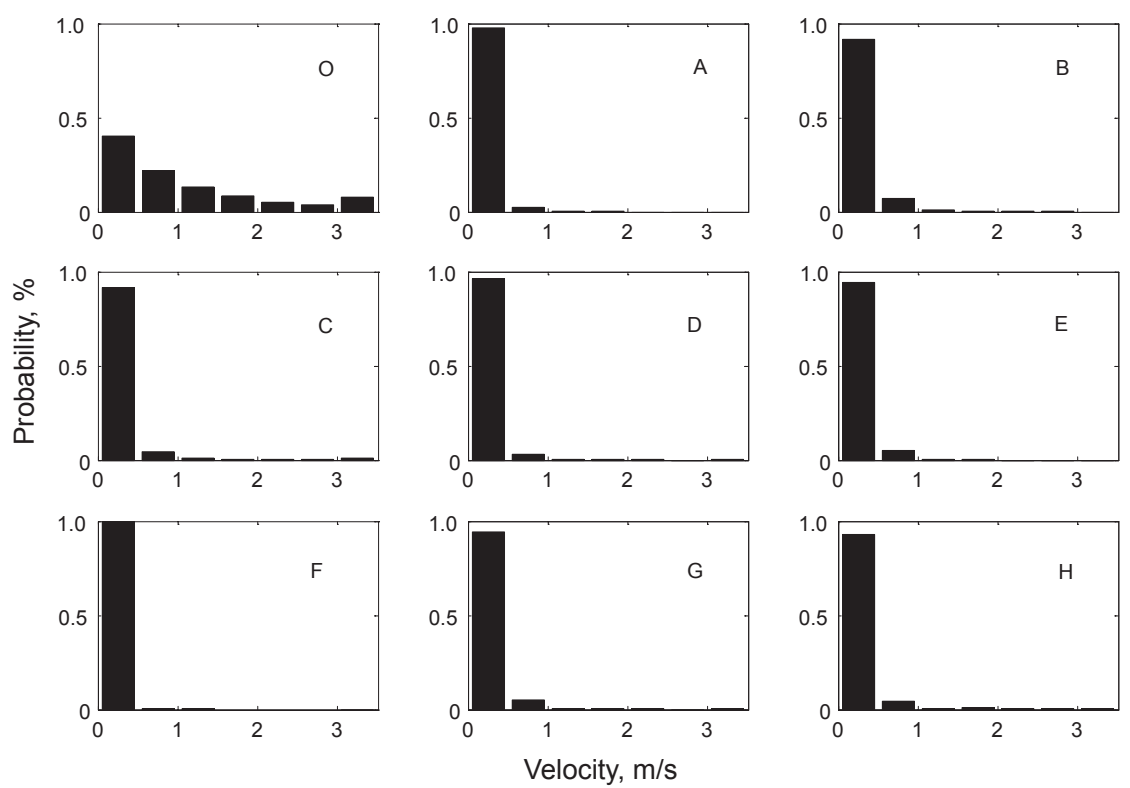

Fig. 7 Probability distribution of particle-wall impact velocity

Therefore setting ribs on the inner wall of the choke can significantly reduce particle average impact velocity, which is a major reason for reducing erosion by setting ribs.

In order to discuss the erosion severity of the choke with different rib geometrical parameters, the relative erosion rate $E / E_{0}$ is defined, namely the ratio of erosion rate of choke wall after setting ribs $E$ and that of before setting ribs $E_{0}$. The smaller the relative erosion rate is, the better the anti-erosion performance. And the relative erosion rates at different rib geometrical parameters are shown in Fig. 8. The mean relative erosion rate of the choke is from 0.04 to 0.61 , with an average value of 0.14 , after setting ribs. That is to say setting ribs can improve the anti-erosion performance of the choke greatly, which show good agreement with the above conclusions. Fig. 8 also indicates that rib type $\mathrm{F}$ has the smallest relative rate, in other words, the best anti-erosion performance can be achieved when there are 4 ribs in the choke, each of them with a height $(H)$ of $3 \mathrm{~mm}$ and a width $(B)$ of $5 \mathrm{~mm}$ spaced 5 mm apart $(L)$.

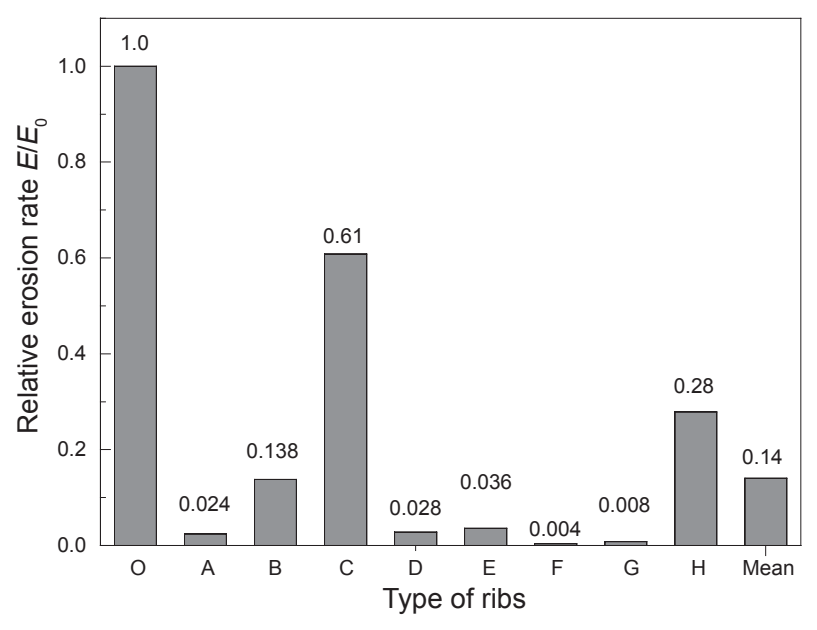

Fig. 8 Relative erosion rate at different rib geometrical parameters

\section{Conclusions}

The following conclusions can be drawn from the above discussion:

1) The discrete particle hard sphere model can simulate the dynamic process of solid particle movements and the non-uniform discrete characteristics of particle distribution in a choke. At the same time, this model can provide detailed information on the impact velocity, impact angle and impact location of an individual particle.

2) Particles accumulate densely in a narrow domain from inlet to outlet of the choke, where the largest particle velocity occurs. The dominating factor affecting particle motion in solid-liquid two-phase flow is the fluid drag force, but particle collisions also play an important role.

3) When the choke has no ribs, only $40 \%$ of particles have a velocity of less than $0.5 \mathrm{~m} / \mathrm{s}$. With ribs in the choke, over $90 \%$ of particles have a velocity of less than $0.5 \mathrm{~m} / \mathrm{s}$. That is to say the average impact velocity of particles lies in the low impact velocity domain after setting ribs.

4) Using ribs can greatly improve the anti-erosion performance of the choke, and the relative best anti-erosion performance can be achieved when setting 4 ribs, each of them with a height $(H)$ of $3 \mathrm{~mm}$ and a width $(B)$ of $5 \mathrm{~mm}$ at 5 mm spacings $(L)$.

\section{Acknowledgements}

This work is supported by the Fund of Innovation Research Group of National Natural Science Foundation of China (Grant NO. 5052160450323001) and Major Program of National Natural Science Foundation of China (Grant No. 50536020).

\section{Reference}

Chen X H, McLaury B, S and Shirazi S A. Numerical and experimental investigation of the relative erosion severity between plugged tees 
and elbows in dilute gas/solid two-phase flow. Wear. 2006. 261 (7-8): 715-729

Crowe C T, Sommerfeld M and Tsuji Y T. Multiphase Flows with Droplets and Particles. Boca Raton: CRC Press. 1998. 112-165

Di Felice R. The voidage function for fluid-particle interaction systems. International Journal of Multiphase Flow. 1994. 20(1): 153-159

Economides M J and Nolte K G. Reservoir Stimulation (3rd Edition). Translated by Zhang B P. Beijing: Petroleum Industry Press. 2002. $133-158$

Elghobashi S. On predicting particle-laden turbulent flows. Applied Scientific Research. 1994. 52(2): 309-329

Fan J R, Luo K, Zhang X Y, et al. Large eddy simulation of the antierosion characteristics of the ribbed-bend in gas-solid flows. Journal of Engineering for Gas Turbines and Power. 2004. 126(3): 672-679

Finnie I. Some reflections on the past and future of erosion. Wear. 1995. 186-187(Part 1): 1-10

Forder A, Thew M and Harrison D. A numerical investigation of solid particle erosion experienced within oilfield control valves. Wear. 1998. 216(2): 184-193

Habib M A and Badr H M. Numerical calculations of erosion in an abrupt pipe contraction of different contraction ratios. International Journal of Numerical Method in Fluids. 2004. 46(1): 19-35

Habib M A, Badr H M, Mansour R B, et al. Erosion rate correlations of a pipe protruded in an abrupt pipe contraction. International Journal of Impact Engineering. 2007. 34(8): 1350-1369

Humphrey J A C. Fundamentals of fluid motion in erosion by solid particle impact. International Journal of Heat and Fluid Flow. 1990. 11(3): 170-195

Jordan K. Erosion in multiphase production of oil \& gas. Corrosion. 1998. Paper No. 058. NACE. 1998

Kang L Q and Guo L J. Eulerian-Lagrangian simulation of aeolian sand transport. Powder Technology, 2006. 162(2): 111-120

Lindsley B A and Marder A R. The effect of velocity on the solid particle erosion rate of alloys. Wear. 1999. 225-229(Part 1): 510-516

McCasland M, Barrilleaux M, Gai H, et al. Predicting and mitigating erosion of downhole flow control equipment in water-injector completions. SPE Annual Technical Conference and Exhibition, 26-29 September 2004, Houston, Texas (SPE 90179)

McLaury B S, Shirazi S A, Shadley J R, et al. Modeling erosion in chokes. ASME Fluids Engineering Division. 1996. 236(1): 773-781

McLaury B S and Wang J. Solid particle erosion in long radius elbows and straight pipes. SPE Annual Technical Conference and Exhibition, 5-8 October 1997, San Antonio, Texas (SPE 38842)

Meng $\mathrm{H}$ and Van der Geld C W M. Particle trajectory computations in steady non-uniform liquid flows. ASME Fluids Engineering Division. 1991. 118: 183-93

Mengütürk M and Sverdrup E F. Calculated tolerance of a large electric utility gas turbine to erosion damage by coal gas ash particles. In: Erosion: Prevention and Useful Application. Edited by Adler W F. Philadelphia: ASTM Spec. Tech. Publ. 1979. 193-2224

Richardson J W, Le K M and Riggs K R. Production casing erosion through annular injection. SPE Annual Technical Conference and Exhibition, 2-5 October 1988, Houston, Texas (SPE 18056)

Song X Q, Lin J Z, Zhao J F, et al. Research on reducing erosion by adding ribs on the wall in particulate two-phase flows. Wear. 1996. 193(1): 1-7

Vincent M C, Miller H B, Milton-Tayler D, et al. Erosion by proppant: a comparison of the erosivity of sand and ceramic proppants during slurry injection and flowback of proppant. SPE Annual Technical Conference and Exhibition, 26-29 September 2004, Houston, Texas (SPE 90604)

Wallace M S, Dempster W M, Scanlon T, et al. Prediction of impact erosion in valve geometries. Wear. 2004. 256(9-10): 927-936

Yao J, Fan J R and Cen K C. Numerical investigation of a new method for reducing bends erosion from particles impacts. Chinese Journal of Chemical Engineering. 2002. 10 (1): 93-101

Zhang Y L. Application and Improvement of Computational Fluid Dynamics (CFD) in Solid Particle Erosion Modeling. Ph.D Thesis. The University of Tulsa. 2006

(Edited by Sun Yanhua) 